\title{
Reduced Synaptic Vesicle Recycling during Hypoxia in Cultured Cortical Neurons
}

\author{
Sergei Fedorovich ${ }^{1}$, Jeannette Hofmeijer ${ }^{2,3}$, Michel J. A. M. van Putten ${ }^{2,4}$ and \\ Joost le Feber ${ }^{2 *}$ \\ ${ }^{1}$ Laboratory of Biophysics and Cellular Engineering, Institute of Biophysics and Cell Engineering, National Academy of \\ Sciences of Belarus, Minsk, Belarus, ${ }^{2}$ Clinical Neurophysiology, University of Twente, Enschede, Netherlands, ${ }^{3}$ Department \\ of Neurology, Rijnstate Hospital, Arnhem, Netherlands, ${ }^{4}$ Department of Neurology and Clinical Neurophysiology, Medisch \\ Spectrum Twente, Enschede, Netherlands
}

OPEN ACCESS

Edited by:

Dirk Schubert,

Donders Institute for Brain Cognition and Behaviour \& Radboud University

Medical Centre, Netherlands

Reviewed by:

Valentina Pasquale,

Italian Institute of Technology, Italy Claire Cheetham,

Carnegie Mellon University, USA

*Correspondence: Joost le Feber

j.lefeber@utwente.n

Received: 24 November 2016 Accepted: 01 February 2017 Published: 16 February 2017

Citation:

Fedorovich S, Hofmeijer J, van Putten MJAM and le Feber J (2017) Reduced Synaptic Vesicle Recycling during Hypoxia in Cultured Cortical Neurons. Front. Cell. Neurosci. 11:32. doi: 10.3389/fncel.2017.00032
Improvement of neuronal recovery in the ischemic penumbra, an area around the core of a brain infarct with some remaining perfusion, has a large potential for the development of therapy against acute ischemic stroke. However, mechanisms that lead to either recovery or secondary damage in the penumbra largely remain unclear. Recent studies in cultured networks of cortical neurons showed that failure of synaptic transmission (referred to as synaptic failure) is a critical factor in the penumbral area, but the mechanisms that lead to synaptic failure are still under investigation. Here we used a Styryl dye, FM1-43, to quantify endocytosis and exocytosis in cultures of rat cortical neurons under normoxic and hypoxic conditions. Hypoxia in cultured cortical networks rapidly depressed endocytosis and, to a lesser extent, exocytosis. These findings support electrophysiological findings that synaptic failure occurs quickly after the induction of hypoxia, and confirms that the failing processes are at least in part presynaptic.

Keywords: synapse, endocytosis, exocytosis, hypoxia, brain, styryl dye, cultured neurons

\section{INTRODUCTION}

Stroke is the third leading cause of death and the second largest cause of chronic adult disability in the Western World. About $85 \%$ concerns a brain infarct, resulting from blockage of arterial blood flow to the brain. In the core of a brain infarct, blood supply is usually not enough to maintain ion gradients across the neuronal plasma membrane, and loss of neuronal function is followed by neuronal death within minutes. Otherwise, in peripheral areas of an infarct, the so called penumbra, damage is not (yet) irreversible due to blood supply from surrounding arteries. In the penumbra, neurons are functionally silent, but structurally intact and viable. These may eventually recover, or proceed to cell death, but the mechanisms behind these diverging scenarios are not clearly understood. Therapies to prevent collateral damage of penumbral brain tissue have a large potential to improve neurological outcome of patients with brain infarcts, but are lacking (George and Steinberg, 2015).

As an early consequence of cerebral ischemia synaptic activity is lost, and failure of synaptic transmission (referred to as synaptic failure) has been proposed to account for electric silence in the penumbra (Hofmeijer and van Putten, 2012; Hofmeijer et al., 2014; le Feber et al., 2016). The changes of synaptic functioning are generally assumed to be reversible (Gao et al., 1999; Bolay et al., 2002; Hofmeijer et al., 2014; le Feber et al., 2016). It is even hypothesized that suppression of functional synaptic activity may reflect a compensatory mechanism that restores the balance between oxygen supply and consumption in favor of maintaining resting potentials and thus 
preserves the neurons' structural integrity (Hochachka et al., 1996). On the other hand, persisting synaptic failure has been associated with progression toward irreversible neuronal damage, even in the absence of membrane depolarization. Most evidence supports the notion that ischemic synaptic failure occurs primarily at the presynaptic terminal and that it involves impaired transmitter release (for a review see Hofmeijer and van Putten, 2012). However, details on the molecular mechanisms are unclear.

Here, we investigated synaptic vesicle recycling, a presynaptic process that seems plausible to play an important role in hypoxia induced synaptic failure. Under normal conditions, synaptic transmission is initiated when an action potential arrives at the presynaptic nerve terminal, inducing the opening of voltage gated $\mathrm{Ca}^{2+}$ channels. The resulting temporary increase of intracellular $\mathrm{Ca}^{2+}$ triggers synapsin phosphorylation, which releases vesicles from the reserve pool to the ready releasable pool to enable exocytosis. After exocytosis, synaptic vesicles are recycled by endocytosis and refilled with neurotransmitter (Dittman and Ryan, 2009). At least several steps of the synaptic vesicle cycle are strongly ATP-dependent, including neurotransmitter loading, disassembling of SNARE complexes, priming, and endocytosis (Südhof, 2004, 2013). It is unknown, what exact parts of the synaptic vesicle cycle are affected by hypoxia. This is difficult to investigate using electrophysiological tools alone. Imaging of styryl dyes (for instance, FM 1-43) allows for separate visualization of various steps of the synaptic vesicle cycle (Cochilla et al., 1999). Here we investigated the effect of hypoxia on endocytosis and exocytosis using the fluorescent dye FM1-43.

\section{METHODS}

\section{Cell Cultures}

We obtained cortical neurons from new-born Wistar rats on the day of birth. All procedures involving animals were conducted according to Dutch and European laws and guidelines, and approved by the Dutch Animal Use Committee (DEC). After trypsin treatment, cells were dissociated by trituration. About 100,000 dissociated neurons were plated on a coverslip or on a multi electrode array (MEA; 60 electrodes with a $30 \mu \mathrm{m}$ diameter and $200 \mu \mathrm{m}$ pitch; Multi Channel Systems, Reutlingen, Germany), both pre-coated with poly ethylene imine (PEI). To enable comparison to earlier electrophysiological measurements, we used the same high cell density as previous work (Hofmeijer et al., 2014; le Feber et al., 2016). Cells were plated at an initial density of approximately 5,000 cells per $\mathrm{mm}^{2}$, with aging, cell densities gradually decreased to $\sim 2,500$ cells $/ \mathrm{mm}^{2}$.

Neurons were obtained in four different preparations and cultured on coverslips $(n=26)$ stored in 24 -well plates or in a circular chamber glued on top of the MEA $(n=2)$. The culture chambers were filled with R12 medium (Romijn et al., 1984) and all cultures were stored in an incubator, under standard conditions of $36^{\circ} \mathrm{C}, 100 \%$ humidity, and $5 \% \mathrm{CO}_{2}$ in air. We kept cells in culture for at least 3 weeks, allowing for the development of mature networks. Medium was refreshed twice a week $(300 \mu \mathrm{L}$ of old medium was replaced by $400 \mu \mathrm{L}$ fresh medium).
For the induction of hypoxia, we placed the cultures under a Plexiglas hood, referred to as hypoxic chamber, where under a continuous flow of a computer regulated mixture of air and nitrogen was kept. Five $\% \mathrm{CO}_{2}$ was added to the gas mixture and humidity was maintained. Cultures on coverslips were put in the hypoxic chamber in 24-well plates, the MEA culture chambers were sealed with watertight but $\mathrm{O}_{2}$ and $\mathrm{CO}_{2}$ permeable foil (MCS; ALA scientific). For experiments, cultures were transferred to a confocal microscope (Zeiss LSM 510). Experiments began after an accommodation period of at least 20 min. Immediately before the start of an experiment covers were removed from the culture chambers to optimize visual access and to facilitate quick medium changes. This allowed oxygen to re-enter the medium, which occurred at a relatively slow rate (see below). From that point, maintenance of sterility was not necessary anymore, because experiments typically lasted less than $10 \mathrm{~min}$.

\section{Hypoxia}

Prior to the measurements, cultures were exposed to hypoxia during $6 \mathrm{~h}$. This was achieved in the hypoxic chamber by replacing $90 \%$ of air by nitrogen, which yielded a lowering of partial oxygen pressure $\left(\mathrm{pO}_{2}\right)$ from $\mathrm{pO}_{2} \approx 160 \mathrm{mmHg}$ to $\mathrm{pO}_{2} \approx 20 \mathrm{mmHg}$. Partial oxygen pressures were measured using an optical oxygen sensor (PHOSPOR, Ocean Optics). Earlier electrophysiological measurements clearly showed synaptic failure during $6 \mathrm{~h}$ of hypoxia at this depth (le Feber et al., 2016). All solutions used for imaging were also kept in the hypoxic chamber to obtain equal $\mathrm{pO}_{2}$, prior to administration. Besides controlled hypoxia, the hypoxic chamber allowed keeping the neurons under standard conditions $\left(36^{\circ} \mathrm{C}, 100 \%\right.$ humidity, and $5 \% \mathrm{CO}_{2}$ ). Control coverslips (without hypoxia) were incubated in a $\mathrm{CO}_{2}$ incubator under standard conditions.

After this incubation period (exposure to hypoxia) the medium of coverslips was changed to medium containing ionotropic receptor inhibitors $50 \mu \mathrm{M}$ DL-2 amino-5phosphonovaleric acid (APV; a selective blocker of the NMDA glutamate receptor; Sigma-Aldrich) and $10 \mu \mathrm{M}$ 6-cyano-7nitroquinoxaline-2,3-dione (CNQX; a selective AMPA receptor blocker; Sigma-Aldrich), which had been exposed to the same hypoxic conditions. Figure 1A illustrates the experimental protocol. In the two cultures plated on MEAs, we recorded spontaneous activity and responses to electrical stimulation as described in le Feber et al. (2016). These cultures were used to verify the efficacy of excitatory blockade at this concentration by evaluation of their responses to electrical stimulation. Two types of imaging solutions were used in different experiments: R12 cell culture medium and the colorless modified Tyrode solutions (136 mM NaCl, $2.5 \mathrm{mM} \mathrm{KCl,} 10 \mathrm{mM}$ HEPES, $10 \mathrm{mM}$ glucose, $1.3 \mathrm{mM} \mathrm{MgCl}_{2}, \mathrm{pH} 7.4$ ), to verify that the color of R12 did not interfere with detection of the FM dye.

\section{Imaging}

Endocytosis and exocytosis were imaged using a fluorescent dye FM1-43 (Sigma-Aldrich) according to Stevens and Williams (Stevens and Williams, 2000) and a confocal microscope (LSM 510, Carl Zeiss) with objective 20x 0.5 n.a. for experiments 


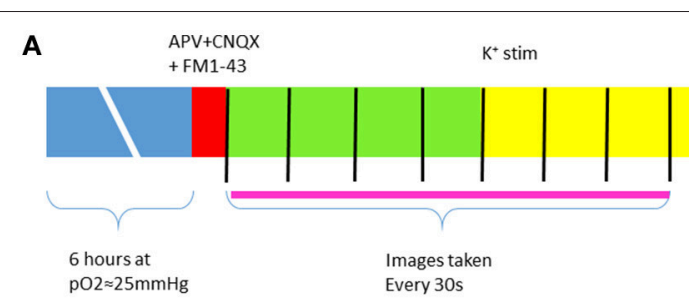

B

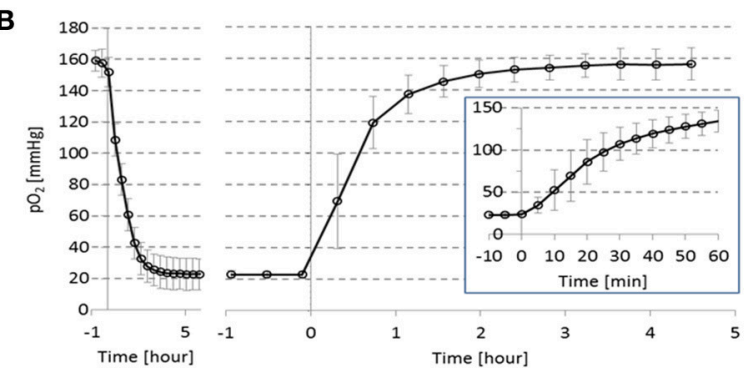

$\mathrm{APV}+\mathrm{CNQX} \quad \mathrm{K}^{+}$stim

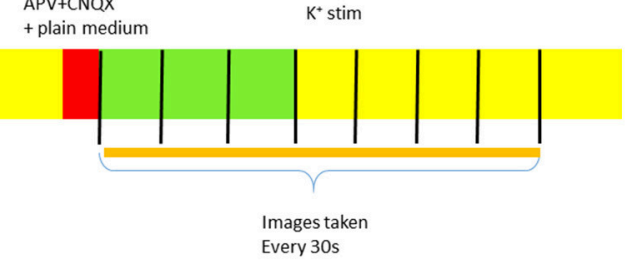

Every 30s
C

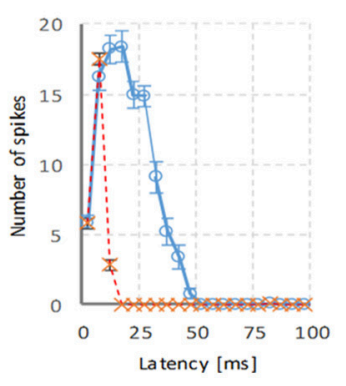

FIGURE 1 | Timeline of the experiments and verification of experimental conditions. (A) Cultures were exposed to $6 \mathrm{~h}$ of hypoxia at $\mathrm{pO} 2 \approx 20 \mathrm{mmHg}$. Then, excitatory synaptic transmission was blocked by APV and CNQX and an FM dye was added to the medium. The cultures were installed under the microscope and imaging started (every $30 \mathrm{sec}$, indicated by vertical black lines). Cultures were stimulated in the 2nd min of imaging by potassium ( $n=26$ ) or electrically ( $n=2$ ). Electrical stimulation was repeated in the 4th min of imaging. Then the FM dye was washed out, and cultures were stimulated again in the 2 nd min after medium change. Endocytosis measurement is indicated by the thick pink line, exocytosis measurement is indicated by the orange line. (B) $\mathrm{Partial}$ oxygen pressure $\left(\mathrm{pO}_{2}\right)$ in culture medium with a cortical culture (mean $\pm S D$ of $n=3$ ) following a stepwise change in gas mixture settings at $t=0$. Left panel: The gas mixture fed to the hypoxic chamber was changed from normoxia $\left(\mathrm{pO}_{2} \approx 160 \mathrm{mmHg}\right)$ to hypoxia $\left(\mathrm{pO}_{2} \approx 20 \mathrm{mmHg}\right)$ at $t=0$. Right panel: At $t=0$ the gas mixture returned to normoxia. Before and after the oxygen measurements, electrical activity was recorded from the neurons, confirming that the cells were alive. Inset: $\mathrm{pO} \mathrm{O}_{2}$ during the $1 \mathrm{st} \mathrm{h}$ at higher temporal resolution. (C) Example of responses to electrical stimulation before ( 0 , solid blue line), or after ( $\mathrm{x}$, dashed red line) blockade of glutamatergic synaptic transmission. All recorded action potentials are counted in $5 \mathrm{~ms}$ bins after each stimulus pulse, mean \pm SEM of 10 stimulus pulses are shown. In both cultures the late phase of the stimulus response (latency >15 ms) was completely blocked at the concentrations used.

with electrical stimulation, or with $40 \mathrm{x} 0.6$ n.a. objective (LD Achroplan, Carl Zeiss) for experiments with potassium stimulation. It was shown that this magnification is sufficient for synaptic vesicles recycling imaging (Klingauf et al., 1998; Stevens and Williams, 2000; Rangaraju et al., 2014). The glass ring on the MEAs impeded larger magnification than $20 \mathrm{x}$ because larger objectives could not get close enough to the sample.

Due to the acute nature of experiments we could not photograph many areas sequentially. Therefore, we used a large scanning area and consequently, the scanning resolution was relatively low (scanning area and scanning resolution are reciprocally related on our confocal, if the total time for imaging is fixed). For quantification of the effect of hypoxia on endo- and exocytosis, reproducibility of changes is crucial. Lower resolution introduces more noise in the images and may thus reduce reproducibility. However, the chosen resolution was sufficient to observe significant differences.

Images were acquired every $30 \mathrm{~s}$. The dye was excited by an argon laser $(488 \mathrm{~nm}$ ) while collecting the emission through a 505 $\mathrm{nm}$ long-pass filter. Two strategies for dye loading and releasing were used in different experiments: electrical and potassium stimulation (see below). In both cases, $10 \mu \mathrm{M}$ of FM1-43 was added to the incubation medium.

The R12 culture medium contains a $\mathrm{pH}$ indicator, and has a slight purple color at healthy $\mathrm{pH}$. To verify that this color did not interfere with imaging, we compared the amplitude of fluorescence changes in R12 with that in modified Tyrode solution, and found no differences (data not shown).

All other images were collected in R12 cell culture medium.

\section{Neuronal Stimulation to Induce Endocytosis and Exocytosis}

All cultures on coverslips were stimulated by adding $60 \mathrm{mM}$ $\mathrm{KCl}$ in the 2 nd min of imaging. If modified Tyrode solution was used, $2 \mathrm{mM}$ of $\mathrm{CaCl}_{2}$ was added in parallel. Before stimulation, there was no fluorescence. Increased fluorescence at $1.5 \mathrm{~min}$ after potassium addition was considered to reflect endocytosis (Gaffield and Betz, 2006). This relatively long period was necessary because endocytosis was relatively slow in our experiments. We could not use short pulses of high potassium concentration due to the absence of a perfusion system. Figure 2B shows a typical response to first $\mathrm{K}^{+}$stimulation to load the dye. Then, medium was changed to wash out the dye and again potassium was added in the 2 nd min of imaging. Decreasing fluorescence during minutes 2-4 after the medium change was considered to reflect exocytosis (see Figure 2C). Background fluorescence in the 2 nd min was set to $100 \%$, and all fluorescence intensities were normalized to this value to facilitate averaging across cultures. Due to the absence of fluorescence before stimulation, such normalization was not possible for the quantification of endocytosis. The 2 cultures on MEAs were electrically stimulated to image endo- and exocytosis, but these results were not used in the current study. 
A

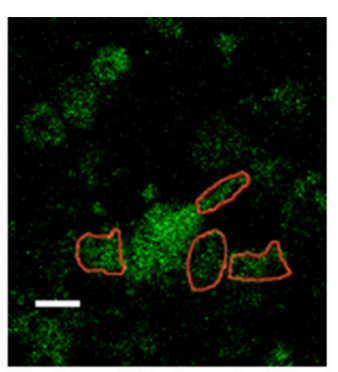

c

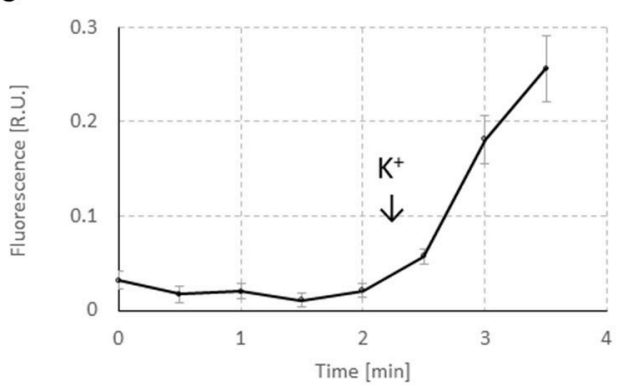

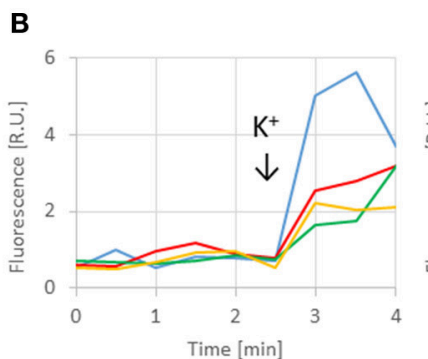
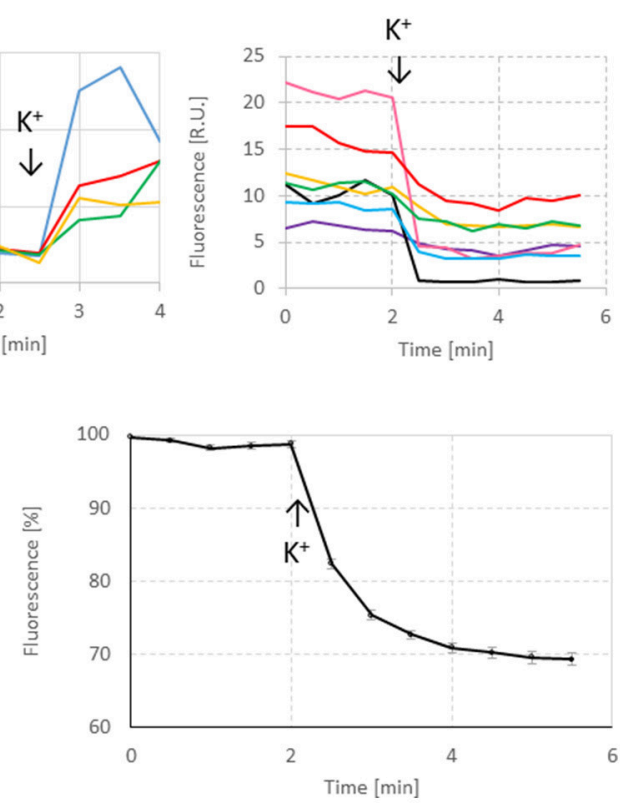

FIGURE 2 | Representative responses of FM1-43 in neurons. (A) Image taken before exocytosis. Red lines indicate selected ROls. White scale bar: 10 um. The imaging resolution is somewhat low, as a consequence of maximizing the scanning area. However, it was sufficient to quantify the effect of hypoxia on endo- and exocytosis and to observe significant differences between normoxia and hypoxia. (B) Representative responses to first potassium stimulation and to second potassium stimulation after washing. First increase in fluorescence was used to quantify endocytosis. The second response (decreased fluorescence) was used to quantify exocytosis. Addition of $60 \mathrm{mM} \mathrm{KCl}$ was indicated by arrows and " $\mathrm{K}^{+}$." (C) Average endocytosis and exocytosis curves under control conditions $(n=258$ ROIs in 11 cultures). For endocytosis curves, minimum fluorescence before stimulation was subtracted from all points to facilitate averaging. Exocytosis curves were normalized to their fluorescence at 2 min before stimulation.

Figure 1A summarizes the experimental protocol.

\section{Analysis}

Images were analyzed using ImageJ. Regions of interest (ROIs) were placed manually around individual puncta or small groups of nearby situated puncta that seemed to respond to stimulation, see Figure 2A. The modus of imaging (endocytosis or exocytosis) was known to the researcher that selected the ROIs, but not the normoxic or hypoxic conditions. Selection of ROIs consisted of three steps.

1. Selection of ROI. In case of endocytosis boutons (group of puncta) with increase of fluorescence were selected, in case of exocytosis boutons with decrease of fluorescence were selected. Very few boutons responded the opposite way (this was only possible in case of exocytosis due to the very low baseline fluorescence in experiments with endocytosis). Locations with unchanging fluorescence were classified as non-specific.

2. Selection of curves. Curves with unstable initial fluorescence were discarded, as well as curves with a "response" onset that differed from the time of stimulation.

3. Selection of amplitudes. Responses that differed more than 2 SD from the averaged response were discarded. Generally, these were boutons with $100 \%$ exocytosis, which most likely were neurons that lysed in response to high potassium.
In all ROIs we calculated the maximum change in fluorescence. Increasing fluorescence during endocytosis is expressed in arbitrary units (R.U.). Minimum fluorescence before stimulation (background fluorescence) fluctuated slightly and was subtracted before averaging across ROIs.

Decreasing florescence during exocytosis is expressed as a fraction of the fluorescence after dye loading to compensate for possible, hypoxia induced, baseline fluorescence differences immediately after loading of the dye.

Data are presented as mean \pm S.E.M unless stated otherwise. Where indicated, statistical significance was evaluated by twotailed Student's $t$-test. Normality of data was confirmed by the Shapiro-Wilkinson test (OriginPro 8).

\section{RESULTS}

Two cultures plated on MEAs were spontaneously active, and showed synchronous patterns, commonly referred to as bursts. In both cultures, electrical stimulation effectively induced a network response before blockade of glutamatergic synaptic transmission, whereas application of APV and CNQX impeded the second, synaptically mediated phase of the response (Figure 1C). Electrical stimulation confirmed the glutamatergic blockade at the used concentrations of APV and CNQX, but could no longer excite a sufficiently large number of neurons, necessary to quantify endocytosis and exocytosis at a network 

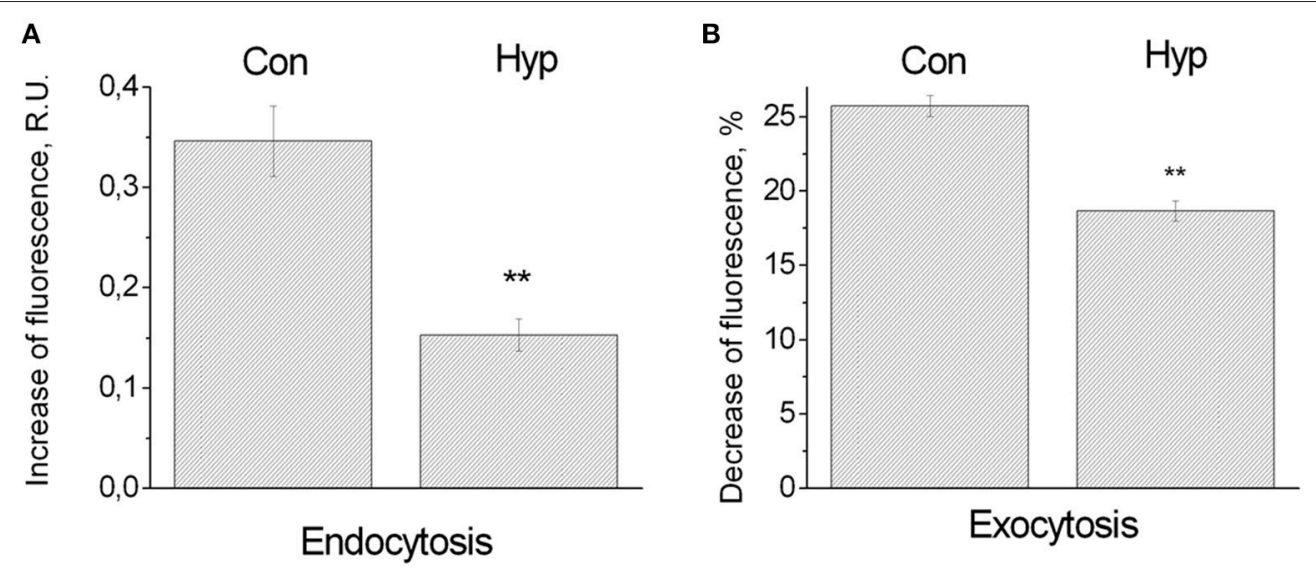

FIGURE 3 | Influence of hypoxia on the synaptic vesicle cycle. (A) Endocytosis. Bars indicate increasing fluorescence upon a series of stimulation induced action potentials, in random units (R.U.), under normoxic conditions (258 ROls in 11 cultures: Con), or hypoxic conditions (101 ROls in 8 cultures: Hyp). Error bars indicate SEM, and reflect differences between ROls. (B) Exocytosis. After loading, extracellular dye was washed out to measure the decreasing fluorescence upon a series of action potentials in response to stimulation. Decreasing fluoresce was normalized to the average fluorescence after dye loading. Error bars indicate SEM and reflect differences between 526 ROls of 14 cultures (control conditions: Con), or 452 ROls in 12 cultures (hypoxic conditions: Hyp). ${ }^{\star *}$ Indicate significant differences $(p<0.01)$.

occurs secondary to impeded endocytosis. Alternatively, energy depletion might directly affect exocytosis, e.g., through reduced phosphorylation of synapsin I, which impedes the transfer of synaptic vesicles from the reserve pool to the readily releasable pool and thus exocytosis. Dual staining for synapsin I and phosphosynapsin may reveal the relevance of this potential mechanism.

Ongoing spontaneous activity might severely hamper quantification of endocytosis and exocytosis. In the current study we used glutamatergic antagonists to block excitatory coupling between the neurons. Consequently, spontaneous activity dropped to (nearly) zero, and occurrence of exo/endocytosis was restricted to the episodes of stimulation. The late (synaptically driven) phase of the stimulus response (latencies $>15 \mathrm{~ms}$ ), but not the early phase $(0-15 \mathrm{~ms})$ was blocked by application of APV (an NMDA receptor antagonist) and CNQX (an AMPA receptor antagonist) (Marom and Shahaf, 2002; Wagenaar et al., 2004). Stimulus responses confirmed the efficacy of the applied blockers (Figure 1C). Although it is common practice in imaging of synaptic vesicle recycling (Stevens and Williams, 2000; Burrone et al., 2006; Orenbuch et al., 2012), the efficacy of electrical stimulation largely dropped due to the application of these blockers. Whereas electrical stimulation under control conditions usually triggered a network burst, after glutamatergic blockade it only directly activated a small subset of neurons. This probably explains the relatively small response to electrical stimulation.

We therefore used potassium to induce depolarization of the plasma membrane and synaptic vesicle recycling. In neurons, the plasma membrane potential is determined by the potassium gradient across the membrane. Therefore, depolarization quickly follows an increase of the extracellular $\mathrm{K}^{+}$concentration. This approach has the advantage that we can investigate the direct influence of hypoxia on synaptic vesicle recycling, avoiding the influence of possible potential changes across the plasma membrane or effects on voltage-gated sodium and potassium channels during shortage of oxygen (Müller and Somjen, 2000; Ayata and Lauritzen, 2015). Initial fluorescence increases in all endocytosis experiments were limited, and could certainly not account for the level of fluorescence at the beginning of subsequent exocytosis experiments, as illustrated in Figure 2. Additional dye was probably endocytosed at the time of washing between endocytosis and exocytosis measurements. During pipetting more dye reached the neurons, which were depolarized by the high potassium. This further increased the FM1-43 fluorescence to the level at which exocytosis measurement started. Still, we used the initial fluorescence increase to compare endocytosis under normoxic and hypoxic conditions. This was possible because this phenomenon occurred under both conditions and we had no reason to believe that it was differently affected by either condition.

Neurotransmitter release depends not only on synaptic vesicle recycling. It also can be dramatically changed by energy depletion induced collapse of resting membrane potential or modification of action potential. Our results clearly show that ATP-dependent steps of synaptic vesicle recycling are involved in synaptic failure during hypoxia. Potentially they can be targets for therapeutic intervention.

\section{ROI Selection}

Manual selection of ROIs is always a bit subjective. However, dye remnants after washing may inadvertently stain the parts of the plasma membrane, or even cell debris. Dye at these locations will respond only in a nonspecific way. Selection of ROIs diminishes the possible contribution of dye at these locations. In addition, selection of ROIs reduces the influence of areas that don't contain boutons. These areas generally reduce fluorescence, as well as fluorescence changes. Thus, they reduce the visualization 
of endo- and exocytosis, and possible alterations induced by hypoxia. Therefore, we tried to position the borders of ROIs closely around boutons (see Figure 2A). Imperfect positioning of the borders might affect the outcome only if areas without boutons were significantly different under hypoxic and control conditions. However, ROI positioning was done blinded to the condition, and given the large number of ROIs per condition it is very improbable that ROIs in one condition contained significantly more areas not containing boutons.

\section{Limitations of Study}

The use of dissociated neurons on multi electrode arrays supports the long term vitality of cultures, but has the disadvantage that cell cultures lack the typical structures found in the in vivo cortex. However, this study focused on generic aspects of synaptic functioning and synaptic vesicle recycling during hypoxia, which does not depend on a specific brain structure.

Another possible limitation lies in the interpretation of hypoxic depth and results from differences between the in vivo and in vitro ranges of normoxia and hypoxia. In vivo studies reported $\mathrm{pO}_{2} \approx 30-35 \mathrm{mmHg}$ during normoxia (Nair et al., 1987; Grote et al., 1996), much lower than normoxia in the current study. Dissociated cortical neurons are usually cultured under high partial oxygen pressures which are interpreted as "normoxic" conditions. It is unclear how this relatively high $\mathrm{pO}_{2}$ relates to the physiological oxygen pressure in vivo. However, minor $\mathrm{pO}_{2}$ drops immediately affected the activity of cultures, even while remaining far above the in vivo normoxia level of 30-35 mmHg (Hofmeijer et al., 2014; le Feber et al., 2016). Furthermore, we chose to restrict the available amount of oxygen but not glucose, whereas circulation problems generally confine

\section{REFERENCES}

Attwell, D., and Laughlin, S. B. (2001). An energy budget for signaling in the grey matter of the brain. J. Cereb. Blood Flow Metab. 21, 1133-1145. doi: 10.1097/00004647-200110000-00001

Ayata, C., and Lauritzen, M. (2015). Spreading depression, spreading depolarizations, and the cerebral vasculature. Physiol. Rev. 95, 953-993. doi: 10.1152/physrev.00027.2014

Bolay, H., Gürsoy-Özdemir, Y., Sara, Y., Onur, R., Can, A., and Dalkara, T. (2002). Persistent defect in transmitter release and synapsin phosphorylation in cerebral cortex after transient moderate ischemic injury. Stroke 33, 1369-1375. doi: 10.1161/01.str.0000013708.54623.de

Burrone, J., Li, Z., and Murthy, V. N. (2006). Studying vesicle cycling in presynaptic terminals using the genetically encoded probe synaptopHluorin. Nat. Prot. 1, 2970-2978. doi: 10.1038/nprot.2006.449

Cochilla, A. J., Angleson, J. K., and Betz, W. J. (1999). Monitoring secretory membrane with FM1-43 fluorescence. Annu. Rev. Neurosci. 22, 1-10. doi: 10.1146/annurev.neuro.22.1.1

Dittman, J., and Ryan, T. A. (2009). Molecular circuitry of endocytosis at nerve terminals. Annu. Rev. Cell Dev. Biol. 25, 133-160. doi: 10.1146/annurev.cellbio.042308.113302

Gaffield, M. A., and Betz, W. J. (2006). Imaging synaptic vesicles exocytosis and endocytosis with FM dyes. Nat. Protoc. 1, 2916-2921. doi: $10.1038 /$ nprot.2006.476

Gao, T. M., Pulsinelli, W. A., and Xu, Z. C. (1999). Changes in membrane properties of CA1 pyramidal neurons after transient forebrain ischemia in vivo. Neuroscience 90, 771-780. doi: 10.1016/S0306-4522(98)00493-X the availability of both. In vivo, additional processes, possibly related to the limited availability of glucose, may occur in parallel to the processes observed in our model system.

In summary, our results demonstrate that hypoxia in cultured cortical networks rapidly depresses synaptic vesicle endocytosis and, to a lesser extent, exocytosis. Blocking of endocytosis has been shown to lead to short-term synaptic depression (Hua et al., 2013). These findings are in agreement with earlier research that showed that synaptic failure occurs quickly after the induction of hypoxia (Hofmeijer et al., 2014; le Feber et al., 2016), and that the failing processes are at least in part presynaptic (Bolay et al., 2002).

\section{AUTHOR CONTRIBUTIONS}

SF: Substantial contributions to conception, cell culture experiments, analysis and interpretation of data, and writing of the manuscript. JH: Contribution to conception, and editing of the manuscript. Mv: Contribution to conception, and editing of the manuscript. JL: Substantial contributions to conception, analysis and interpretation of data, project management, and writing of the manuscript.

\section{ACKNOWLEDGMENTS}

The authors would like to thank Dr Gerco Hassink and Stef Regeling for the preparation of cultures. This study was supported by the Netherlands Organization for Scientific Research, NWO (Grant number 040.11.479)
George, P. M., and Steinberg, G. K. (2015). Novel stroke therapeutics: unraveling stroke pathophysiology and its impact on clinical treatments. Neuron 87, 297-309. doi: 10.1016/j.neuron.2015.05.041

Grote, J., Laue, O., Eiring, P., and Wehler, M. (1996). Evaluation of brain tissue $\mathrm{O}_{2}$ supply based on results of $\mathrm{PO}_{2}$ measurements with needle and surface microelectrodes. J. Auton. Nerv. Syst. 57, 168-172. doi: 10.1016/0165-1838(95)00096-8

Heidelberger, R., Sterling, P., and Mathews, G. (2002). Roles of ATP in depletion and replenishment of the releasable pool of synaptic vesicles. J. Neurophysiol. 88, 98-106.

Hochachka, P. W., Buck, L. T., Doll, C. J., and Land, S. C. (1996). Unifying theory of hypoxia tolerance: molecular/metabolic defense and rescue mechanisms for surviving oxygen lack. Proc. Natl. Acad. Sci. U.S.A. 93, 9493-9498. doi: 10.1073/pnas.93.18.9493

Hofmeijer, J., Mulder, A. T., Farinha, A. C., vanPutten, M. J. A. M., and le Feber, J. (2014). Mild hypoxia affects synaptic connectivity incultured neuronal networks. Brain Res. 1557, 180-189. doi: 10.1016/j.brainres.2014.02.027

Hofmeijer, J., and van Putten, M. J. A. M. (2012). Ischemic cerebral damage. Stroke 43, 607-615. doi: 10.1161/STROKEAHA.111.632943

Hrynevich, S. V., Waseem, T. V., Hebert, A., Pellerin, L., and Fedorovich, S. V. (2016). beta-Hydroxybutyrate supports synaptic vesicle cycling but reduces endocytosis and exocytosis in rat brain synaptosomes. Neurochem. Int. 93, 73-81. doi: 10.1016/j.neuint.2015.12.014

Hua, Y., Woehler, A., Kahms, M., Haucke, V., Neher, E., and Klingauf, J. (2013). Blocking endocytosis enhances short-term synaptic depression under conditions of normal availability of vesicles. Neuron 80, 343-349. doi: 10.1016/j.neuron.2013.08.010 
Klingauf, J., Kavalali, E. T., and Tsien, R. W. (1998). Kinetics and regulation of fast endocytosis at hippocampal synapses. Nature 394, 581-585. doi: 10.1038/29079

le Feber, J., Tzafi Pavlidou, S., Erkamp, N., van Putten, M. J. A. M., and Hofmeijer, J. (2016). Progression of neuronal damage in an In Vitro model of the Ischemic, Penumbra. PLoS ONE 11:e0147231. doi: 10.1371/journal.pone.0147231

Marom, S., and Shahaf, G. (2002). Development, learning and memory in large random networks of cortical neurons: lessons beyond anatomy. Q. Rev. Biophys. 35, 63-87. doi: 10.1017/S0033583501003742

Müller, M., and Somjen, G. G. (2000). $\mathrm{Na}^{+}$dependence and the role of glutamate receptors and $\mathrm{Na}^{+}$channels in ion fluxes during hypoxia of rat hippocampal slices. J. Neurophysiol. 84, 1869-1880.

Nair, P. K., Buerk, D. G., and Halsey, J. H. Jr. (1987). Comparisons of oxygen metabolism and Tissue pO2 in cortex and hippocampus of Gerbil Brain. Stroke 18, 616-622. doi: 10.1161/01.STR.18.3.616

Orenbuch, A., Shalev, L., Marra, V., Sinai, I., Lavy, Y., Kahn, J., et al. (2012). Synapsin selectively controls the mobility of resting pool vesicles at hippocampal terminals. J. Neurosci. 32, 3969-3980. doi: 10.1523/jneurosci.5058-11.2012

Pathak, D., Shields, L. Y., Mendelsohn, B. A., Haddad, D., Lin, W., Gerencser, A. A., et al. (2015). The role of mitochondrially derived ATP in synaptic vesicle recycling. J. Biol. Chem. 290, 22325-22336. doi: 10.1074/jbc.m115. 656405

Rangaraju, V., Calloway, N., and Ryan, T. A. (2014). Activity-driven local ATP synthesis is required for synaptic function. Cell 156, 825-835. doi: 10.1016/j.cell.2013.12.042

Romijn, H. J., van Huizen, F., and Wolters, P. S. (1984). Towards an improved serum-free, chemically defined medium for long-term culturing of cerebral cortex tissue. Neurosci. Biobehav. Rev. 8, 301-334. doi: 10.1016/0149-7634(84)90055-1

Stevens, C. F., and Williams, J. H. (2000). "Kiss and run" exocytosis at hippocampal synapses. Proc. Natl. Acad. Sci. U.S.A. 97, 12828-12833. doi: $10.1073 /$ pnas. 230438697

Südhof, T. C. (2004). The synaptic vesicle cycle. Annu. Rev. Neurosci. 27, 509-547. doi: 10.1146/annurev.neuro.26.041002.131412

Südhof, T. C. (2013). Neurotransmitter release: the last millisecond in the life of a synaptic vesicle. Neuron 80, 675-690. doi: 10.1016/j.neuron.2013.10.022

Sun, M.-K., Xu, H., and Alkon, D. L. (2002). Pharmacological protection of synaptic function, spatial learning, and memory from transient hypoxia in rats. J. Pharmacol. Exp. Ther. 300, 408-416. doi: 10.1124/jpet.300.2.408

Wagenaar, D. A., Pine, J., and Potter, S. M. (2004). Effective parameters for stimulation of dissociated cultures using multi-electrode arrays. J. Neurosci. Methods 138, 27-37. doi: 10.1016/j.jneumeth.2004.03.005

Conflict of Interest Statement: The authors declare that the research was conducted in the absence of any commercial or financial relationships that could be construed as a potential conflict of interest.

Copyright (c) 2017 Fedorovich, Hofmeijer, van Putten and le Feber. This is an openaccess article distributed under the terms of the Creative Commons Attribution License (CC BY). The use, distribution or reproduction in other forums is permitted, provided the original author(s) or licensor are credited and that the original publication in this journal is cited, in accordance with accepted academic practice. No use, distribution or reproduction is permitted which does not comply with these terms. 